\title{
Methodologies for the Synthesis of Reliable MEA Electrical Power System Architectures
}

\author{
Angel Recalde \\ University of Nottingham \\ Institute for Aerospace Technology \\ Nottingham, United Kingdom \\ angel.recalde@nottingham.ac.uk
}

\author{
Serhiy Bozhko \\ University of Nottingham \\ Institute for Aerospace Technology \\ Nottingham, United Kingdom \\ serhiy.bozhko@nottingham.ac.uk
}

\author{
Jason Atkin \\ University of Nottingham \\ School of Computer Science \\ Nottingham, United Kingdom \\ jason.atkin@nottingham.ac.uk
}

\begin{abstract}
This paper reviews the main optimization approaches and design frameworks found in the literature for the synthesis of power system architectures for More Electric Aircraft (MEA). System- and network- engineering are the main interdisciplinary fields that have been used to conceive new highly reliable architectural solutions that demands both disruptive technologies utilization and appropriate complexity management. Being safety the most important feature, it is imperative to explore the benefits these design approaches can offer in the formulation of a MEA power system design problem considering safety and reliability requirements.
\end{abstract}

Keywords-Aircraft power system architecture, More Electric Aircraft, network design, system reliability, reliability-based design

\section{INTRODUCTION}

The MEA is a concept that defines the future of aircraft electrification. Global commitments for achieving a more sustainable environment have triggered several agreements committed to greatly reduce the amount of fossil fuel emissions. Given that the aircraft industry contributes around $3 \%$ to the total world's emissions [1], and this figure will definitely increase in the next decades, it is imperative to explore aircraft electrification as the key enabler for cleaner skies, emission reduction, and higher efficiencies [2], [3]. Certainly, electrical power system (EPS) plays a vital role in achieving aircraft electrification because energy conversion on-board will be redefined. With the production and commissioning of aircraft electrification flagbearers such as Boeing B787 and Airbus A380, transformation of hydraulicand pneumatic- systems into more efficient electromechanical counterparts have become a requisite for improving overall performance while complying with rigorous reliability requirements. Being a safety-driven industry, the future MEA EPS will have to comply with the strictest safety and reliability specifications.

Today's aircraft power systems are divided in left- and right- sides, each one with their own electrical generation, auxiliary systems, and loads. Because of this configuration, it can be split into two autonomous microgrids with the possibility of reconfiguration whenever contingencies occur, such that safety is never compromised. Airworthiness specifications rule aerospace engineering in this matter and provides all the design guidelines to produce a reliable system [4]. Due to the adoption of new technologies and the integration of more-electric subsystems, known as fly-by-wire, the EPS will require to maintain or even exceed current reliability specifications due to the introduction of new topologies, devices and contingency types. In this context, the exploration of a broader design space, sometimes even unfamiliar with respect to the current state-of-the-art in aircraft engineering, could lead to innovative solutions for the MEA EPS architecture. Hence, it is imperative to examine both the design approaches and standards that will guide the synthesis of new architectures for MEA EPS. However, reliability- and performance- based optimization formulations will follow the industry driver's requirements to ensure feasibility. The rest of the paper is organized as follows. Section II describes the implications of reliability in the design of a MEA EPS. In Section III, the predominant reliability oriented design approaches are explored and discussed. Finally, the paper comes to its conclusion in Section IV.

\section{RELIABILITY IN THE MEA EPS DESIGN}

Safety is the condition of being secured from danger or disabled to cause danger. In order to assess the degree of safety, systems comply with a certain level of reliability. Safety can also be defined by other attributes such as robustness, resiliency, and survivability. Depending on the application, these attributes are defined on a set of functional and safety requirements. For instance, an EPS has survivability if they can supply critical loads under any failure condition [5], or it is resilient if it maintains the healthy operation of un-compromised circuits after a catastrophic event. In the MEA EPS design context, safety attributes can be defined as in Fig. 1 in which there are two qualifiers: failure recurrence, and failure impact.

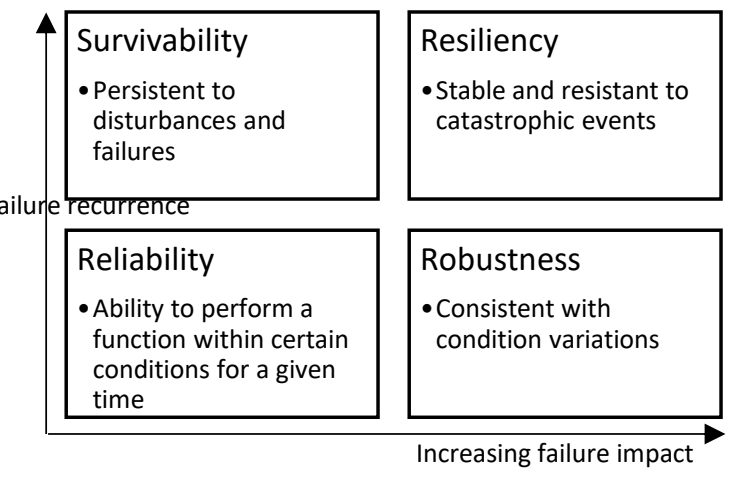

Fig. 1. MEA EPS's safety attributes conceptual map

Aerospace engineering design have consistently focused on reliability, which includes survivability, resiliency, and robustness indistinctly. However, in addition to compliance to these attributes, a safer and more reliable MEA EPS also relies on economic feasibility. Opportunities for regional markets face conflicting requirements, quicker mission deployments, new technology adoption that requires substantial training or additional power-by-the-hour contracts, increased complexity in systems' integration, faster time-to-market requirements, and cost-effective business case implementations. On one hand, conventional design has proven to optimize the system by optimizing each component separately yet observing 
reliability specifications. On the other hand, future designs pose an engineering challenge for which current tools are left unprepared. In the past, severe accidents due to unexpected failures have not been oblivious to the aircraft industry. Thus, reliability and safety are of utmost importance in the MEA design, and this is why future design frameworks has the challenge to provide solutions that exceed today's performance, regardless of their level of complexity. With such premise, there is no room for error. In an attempt to depart from conventional design methods, MEA EPS design has revisited system's engineering principles and network design formulations. In any case, a reliability-based optimization has served as a tool that provides the mathematical framework to explore the design space to construct an optimal architecture. For the rest of the paper, reliability is used to define the degree of safety of a MEA EPS. The implications of conventional design flow and the aerospace design guidelines are outlined shortly.

\section{A. Conventional Design}

The main structure of conventional design (CD) consists of an iterative process where unsatisfied performance evaluation generates new specifications as shown in Fig. 2, until it achieves the expected performance.

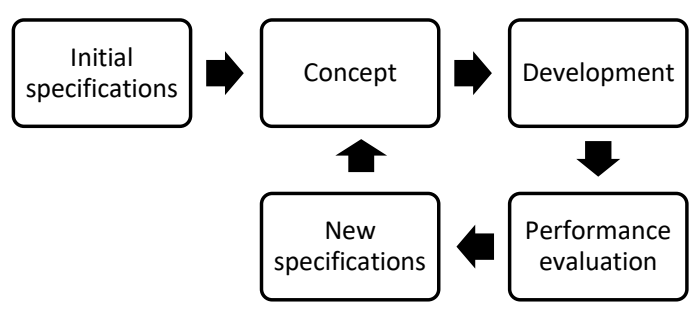

Fig. 2. Conventional Design (CD)

A common application of CD is sub-system design, with very high reliability solutions. However, with the increase in MEA complexity, CD could be time- and resourceconsuming, especially when driven by a large number of conflicting specifications. Besides, limiting the investigation of innovative and disruptive solutions is likely because uncertainty forbids diversion from known designs. The exploration of new EPS architectures for MEA demands a novel construction process that merges design and performance evaluation in an integrated manner, unlike the iterative locally-optimized CD's method, with careful attention to smart utilization of constraint resources (weight, volume, cost) for optimal power density and efficiency. An efficient way to explore the MEA EPS requires the adoption of system engineering and network design approaches, as shown in Fig. 3. On one hand, network design and graph theory allows the definition of an interconnected structure for generators-to-loads power transfer. On the other hand, system engineering provides the principles to integrate and manage complexity and performance evaluation over the system's life cycle. In recent years, novel design frameworks based on the integration of performance evaluation and the design space search have produced MEA prototypes, with the potential of producing accelerated time-to-market developments and synthesizing innovative architectures.

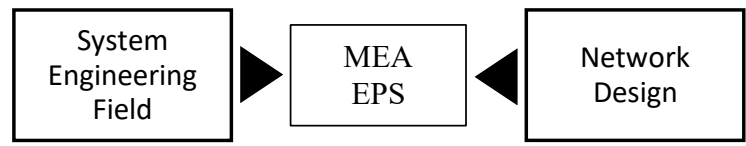

Fig. 3. Field contributions for MEA EPS design

\section{B. Aerospace Design Guidelines}

In the aircraft industry, designs must comply with military and aerospace standards and guidelines, which are also followed by commercial aviation. MIL-STD-217 and other reliability standards shown in TABLE I. are mandatory for aircraft electrical systems certification. However, these standards will experience profound transformations to reflect new functional and operational requirements because the exploration of future MEA will lead to radical different topologies and technological innovations [6].

TABLE I. RELIABILITY STANDARDS AND GUIDELINES FOR ELECTRICAL SYSTEMS

\begin{tabular}{|c|c|}
\hline Standard & Description \\
\hline $\begin{array}{c}M I L-H D B K-217 \\
M I L-S T D-217\end{array}$ & $\begin{array}{l}\text { Developed by the US DoD for military and } \\
\text { aerospace. It is used as a reliability prediction } \\
\text { methodology. The Stress Analysis section is } \\
\text { applied on different operating conditions } \\
\text { (environment, temperature, voltage, current, and } \\
\text { power levels). Latest version: } 217 \mathrm{~F}, 2^{\text {nd }} \text { rev. } 1995\end{array}$ \\
\hline$M I L-S T D-1553$ & $\begin{array}{l}\text { Standard for data buses that allows electronic sub- } \\
\text { systems to interact with each other and other on- } \\
\text { board flight computers. }\end{array}$ \\
\hline$S A E-A R P-4761$ & $\begin{array}{l}\text { Used to determine compliance with Federal } \\
\text { Aviation Regulations (System Safety Assessment). } \\
\text { Primary analyses: Reliability Prediction, FMEA } \\
\text { and FTA, Common Cause Analysis, and Failure } \\
\text { Mode and Effects Summary. }\end{array}$ \\
\hline$S A E-A R P-4754$ & $\begin{array}{l}\text { Guideline developed by SAE International that } \\
\text { addresses the development processes that support } \\
\text { the certification of aircraft systems. }\end{array}$ \\
\hline
\end{tabular}

\section{MEA RELIABILITY ORIENTED DESIGNS}

The MEA EPS design comprises not only the architecture generation, i.e. topology structure, but also the performance evaluation, being reliability the main performance index. According to IEC 60050-191, system's reliability is the probability of performing the system's function all over a time interval $t$. This concept dominates reliability oriented approaches have been used in the design of MEA EPS. TABLE II. presents a survey on recent design approaches and applications for MEA EPS found in the literature.

\section{A. System Engineering Approach}

System's design defines a structure that satisfies a number of functional specifications based on the expected system's performance. Given a set of reliability specifications, system's design becomes a collection of methodologies intended to maximize reliability targets, hence, mathematical optimization is the key enabler. Recently, MEA EPS synthesis has been assessed either with unified framework approaches (UF) or reliability-based design optimization techniques (RBDO), as shown in Fig. 4. It is relevant to mention that Platform Based Design (PBD), a method originated in the electronic devices industry, has been introduced in the MEA EPS design with strong adoption of new performanceverification' tools and novel specification languages. Although PBD has been applied in micro-electronics systems design due to its high complexity and large scale integration (millions of components), it has also been applied to the electric power system design with two main approaches: Correct by Construction $(\mathrm{CbC})$ and Contract based design (CbD). The latter has included controllability performance evaluation within the design process. 


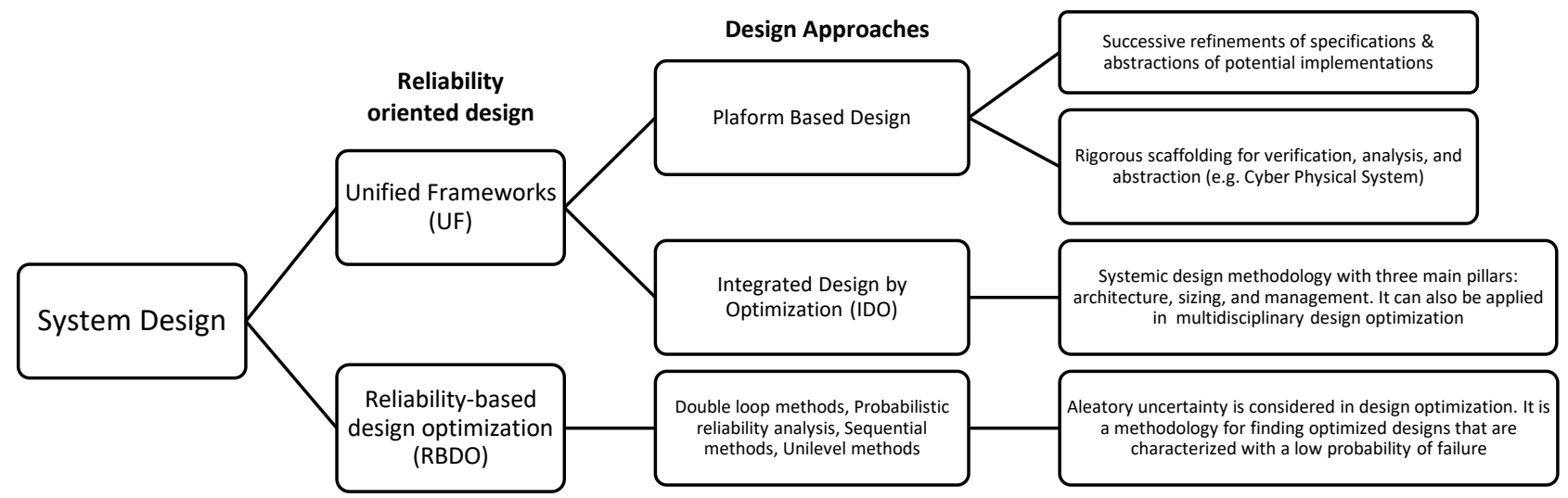

Fig. 4. Reliability-oriented approaches for system design

In [7], CbC develops some mathematical equivalences for functional and reliability specifications in 4 steps or platforms: 1) generation selection and generator-load pairing, 2) power distribution design based on previous generator selection solution, 3) control system synthesis, and 4) embedded system design. Each platform constitutes a high level optimized solution whose proximity to physical implementation depends on the platform's abstraction itself and the number of refinement steps. Optimization in $\mathrm{CbC}$ has utilized Mixed Integer Linear Programming (MILP) formulations due to its convergence to a global optimum and the availability of efficient commercial solvers. The main objective performs trade-off between weight and cost minimization, with reliability and efficiency maximization. The control and embedded systems design are still object of investigation. On the other hand, $\mathrm{CbD}$ uses contracts to translate high-level functional specifications into detailed performance descriptions, e.g. exact propositions that are satisfiable and verifiable [8], [9]. CbD has been applied in the synthesis of MEA EPS architecture (topology) and the definition of its control system [5]. The cost of integrating performance evaluation within the design has been the introduction of new computational tools and mathematical languages explicitly adapted from the computer science field into the aerospace design area. In [5], Linear- and Signal- Temporal Logic (LTL/STL) are used as formal languages to describe in detail the functional specifications within a minimum cost design assessment. System's reliability is measured as a probability of failure (not being able to supply critical loads), which is evaluated with an Integer Programming modulo approach [10]. LTL has required the use of advanced computational synthesis tools, such as the reactive protocols synthesizer SR(1) [8]. Following this line, applications for MEA EPS have been reported in [9], [11] with some testbeds developed on TULIP [12]-[14] which has been implemented in CAD with the JTLV verification scripting environment [15]. Further plans to investigate automatic generation of contracts and contract language definition [5] are expected [16]. CbD presents a rigorous methodology for the design of MEA EPS consisting in three main stages: 1) topology synthesis, 2) control synthesis, and 3) simulation-based design exploration and verification. $\mathrm{CbD}$ and $\mathrm{CbC}$ have been part of Cyber Physical Systems initiatives for MEA designs, including realtime performance assessment [17].

TABle II. Design Methodologies Applied on MEA Electric Power System

\begin{tabular}{|c|c|c|}
\hline Methodology & Approaches & MEA Design Application \\
\hline \multirow{2}{*}{$\begin{array}{l}\text { Platform } \\
\text { Based Design } \\
\quad(P B D)\end{array}$} & $\begin{array}{l}\text { Contracts for } \\
\text { System Design } \\
\text { CbD [18] }\end{array}$ & $\begin{array}{l}\text { Contract-based methodology for aircraft power system design [5], architecture synthesis for aircraft } \\
\text { power systems [19], contract based design for cyber-physical systems [20]. }\end{array}$ \\
\hline & $\begin{array}{l}\text { Correct-by- } \\
\text { construction } \\
\mathrm{CbC}\end{array}$ & $\begin{array}{l}\text { Correct-by-Construction Design of Aircraft Electric Power Systems [7], Platform-Based Design for } \\
\text { Aircraft Electric Power Systems [21], Synthesis of reliable cyber-physical architectures [10],[22], } \\
\text { Managing complexity in aerospace systems [23]. }\end{array}$ \\
\hline \multicolumn{2}{|c|}{$\begin{array}{l}\text { Integrated Design by Optimization } \\
\text { (IDO) }\end{array}$} & $\begin{array}{l}\text { Opportunities on coupling new architectures and technologies to optimize integrated power sub- } \\
\text { systems [24], Several design objectives involving numerous areas of progress in terms of power } \\
\text { integration at both the component and device level [3], Design methodology based on a systemic } \\
\text { viewpoint [25], A system level integrated design by optimization is explored on MEA [26]. }\end{array}$ \\
\hline \multicolumn{2}{|c|}{ Conventional Design Flow (CDF) } & $\begin{array}{l}\text { There have been applications for aircraft design project management [27]. Some reliability tools for } \\
\text { the design process are Failure Mode Effects (FMEA, FMES), Monte Carlo Simulation, and Fault } \\
\text { Tree Analysis (FTA), Highly Accelerated Life Tests (HALT), Highly Accelerated Stress Screening } \\
\text { (HASS), and Accelerated Life Test (ALT) [4]. }\end{array}$ \\
\hline \multicolumn{2}{|c|}{$\begin{array}{l}\text { Reliability Based Design } \\
\text { Optimization (RBDO) }\end{array}$} & $\begin{array}{l}\text { Most of the applications are for civil and structural engineering [28], with rare attempts in electrical } \\
\text { networks [29]. The 1st-order- and 2nd-order methods FORM \& SORM are well known in } \\
\text { structural engineering design [30]. }\end{array}$ \\
\hline $\begin{array}{l}\text { Other } \\
\text { optimization- } \\
\text { based methods }\end{array}$ & $\begin{array}{l}\text { Multi-objective } \\
\text { optimization, } \\
\text { higher } \\
\text { complexity } \\
\text { methods with } \\
\text { reliability, } \\
\text { multidisciplinary } \\
\text { optimization }\end{array}$ & $\begin{array}{l}\text { Architectural options: MEA topology options [31],[32], system architecture for adaptability [33], } \\
\text { power conversion modelling based on building blocks models [34], design simplification based on } \\
\text { high level of abstraction simulation models [35]. } \\
\text { Improvements of current electrical performance: reconfiguration and balancing problem in aircraft } \\
\text { AC networks (Airbus operations) [36], weight saving optimized distribution system with modern } \\
\text { concepts (Airbus operations) [37], optimization of energy-supply-structure of modern aircraft } \\
\text { utilizing conventional power system technologies [38]. } \\
\text { Exploration of new architectures: exploration of several HVDC-architectures for aircraft applications } \\
\text { (Airbus operations) [32], Ptolemy II-Metropolis/Metro II tools for architectural explorations (IBM, } \\
\text { UT, Bosch, NI, Toyota) [39], analysis of HVDC parallel electric power system for MEA [40]. }\end{array}$ \\
\hline
\end{tabular}


Still, there is an enormous potential to explore MEA EPS with novel design frameworks considering the integration of computation, communication, and control into a highly integrated system.

\section{B. Network Design Problem}

In the current stage of MEA EPS early development, interest in network flow and graph theory have increased dramatically. Power balance and other performance constraints based on power or energy flow are linear, which is the reason why MILP is amenable to network design formulations. The EPS is a network whose purpose is to route electrical energy from generators (sources) to loads (sinks) in a reliable and efficient manner. Network design fits adequately as a synthesizer of an EPS network because it can formulate a network construction problem [41]. Network design has been extensively used in the planning and operation of transmission and distribution EPS [42]. A classification of network design approaches according to the problem's objective is shown in Fig. 5. It is relevant to highlight the fact that resiliency and survivability constraints can be seamlessly added to a network problem without the necessity of a separate performance analysis. This provides an efficient way to integrate reliability performance evaluation. For instance, system's survivability to any single-component failure event ( $N-1$ rule) can be modelled as a constraint in which given a set of failure states, the distribution system provides sufficient paths for generators to reach the critical loads.

\begin{tabular}{|c|c|}
\hline $\begin{array}{l}\text { Number of } \\
\text { commodities }\end{array}$ & - Single- or Multi-commodity problem \\
\hline Route definition & - Path- or Arc- based \\
\hline $\begin{array}{l}\text { Infraestructure } \\
\text { limitations }\end{array}$ & - Capacitated or uncapacitated \\
\hline Route cost & $\begin{array}{l}\text { - Fixed-charge network flow } \\
\text { - Generalized fixed-charge network flow }\end{array}$ \\
\hline Reliability & $\begin{array}{l}\text { - Resilient network design } \\
\text { - Survivable network design }\end{array}$ \\
\hline
\end{tabular}

Fig. 5. Network Design according to problem formulation

\section{Other Reliability-based Design Approaches}

Apart from the PBD inspired and the network design approaches, Integrated Design by Optimization (IDO) focuses on the use of model-based analysis with advanced simulation tools [24], [26]. IDO consists in a group of techniques, algorithms, and optimization tools that enables integrated performance evaluation in the design of complex systems via advanced model-based description of components. This representation involves a high number of inter-element and inter-domain couplings, and system-mission interactions, i.e. system as a multi-disciplinary equipment. The IDO's design process consists in linking the system's model to an optimization algorithm with multiple decision criteria such as geometry, efficiency, and dynamic performance. These criteria are optimized to accomplish quality, stability, and compatibility (electrical, thermal, magnetic, etc.). In [25], there are several IDO-based methods to manage data processing, sizing, multi-level, and multi-criteria design in electrical energy systems. The formal analysis, synthesis, and management methods used in IDO are presented as systemic design methodologies in [43]. IDO reports MEA EPS generation system optimization efforts and other electromechanical actuator sub-systems designs in [24].

In addition to IDO, Reliability-Based Design Optimization (RBDO) is a tool that has been extensively applied in civil and structural engineering, albeit seldom in MEA EPS. TABLE II. enumerates some applications of RBDO in structural analysis and design. The main purpose of RBDO is to consider aleatory (random) uncertainty in design optimization. In most cases, reliability requirements are stated as hard constraints in an optimization problem, each constraint related to a given failure mode and its probability of failure. There have been very limited applications of RBDO in electrical engineering, e.g. attempts in [29], [44] introduced probabilistic constraints in an EPS design optimization problem, while in [45] a piezoelectric energy harvester is designed. However, beyond these applications no other approach in EPS has been reported. Although this methodology could aim in developing a reliable design, it lacks of the flexibility to integrate the majority of functional control requirements explicitly. Further to IDO, and RBDO approaches, there have been other contributions in optimizing existing EPS architectures in order to improve specific attributes. For instance, [36], [37] performed research to optimize current AC three-phase aircraft infrastructure, and also explored HVDC configurations for future MEA [32]. Although not adopting rigorous frameworks such as PBD or RBDO, these methodologies are still reliability-oriented. Other efforts have been directed towards investigating MEA pre-defined architecture selection [46], adaptability under different operational scenarios [33], candidate architecture analysis via stability performance [47], among other technologies adoption and complexity management [2].

In summary, MEA EPS design is being shaped by a number of framework and techniques that, although unfamiliar to in the synthesis of new architectures, have shown advantageous in managing increasing complexity, innovative technologies, and reliable topologies.

\section{CONCLUSIONS}

A brief discussion on the existing contributions for the design of novel architectures for future MEA power systems considering reliability has been presented. Due to the increasing complexity of the technology that will become part of MEA, it is necessary to combine efforts between several engineering fields to produce an efficient design framework that needs to depart from conventional design if integration challenges must be faced. PBD, Cyber Physical Systems, IDO, and other optimization based approaches places MILP as an efficient tool to achieve an optimized solution. Given the early stage in which MEA EPS design resides, there is still potential to introduce innovative methods in MEA.

\section{ACKNOWLEDGMENT}

This work is funded by the INNOVATIVE doctoral programme. The INNOVATIVE programme is partially funded by the Marie-Curie Initial Training Networks (ITN) action (project number 665468), and partially by the Institute for Aerospace Technology (IAT) at the University of Nottingham.

\section{REFERENCES}

[1] N. A. of Sciences Engineering and Medicine and C. on Propulsion, Commercial Aircraft Propulsion and Energy Systems Research. Washington, D.C.: National Academies Press, 2016.

[2] P. Wheeler and S. Bozhko, "The more electric aircraft: Technology 
and challenges," IEEE Electrification Magazine, vol. 2, no. 4, pp. 612, Dec-2014.

[3] X. Roboam, B. Sareni, and A. De Andrade, "More Electricity in the Air," Industrial Electronics Magazine, IEEE, vol. 6, no. 4, pp. 6-17, Dec-2012.

[4] V. Crk, "Reliability Engineering Challenges in Aerospace Industry," 2011.

[5] P. Nuzzo et al., "A contract-based methodology for aircraft electric power system design," IEEE Access, vol. 2, pp. 1-25, 2014

[6] G. Buticchi, S. Bozhko, M. Liserre, P. Wheeler, and K. Al-Haddad, "On-board microgrids for the more electric aircraft - Technology review," IEEE Trans. Ind. Electron., vol. 66, no. 7, pp. 5588-5599, 2019.

[7] A. Pinto, S. Becz, and H. Reeve, "Correct-by-Construction Design of Aircraft Electric Power Systems," in 10th AIAA Aviation Technology, Integration, and Operations (ATIO) Conference, 2010, pp. 1-11.

[8] R. Bloem, B. Jobstmann, N. Piterman, A. Pnueli, and Y. Sa'Ar, "Synthesis of Reactive(1) designs," J. Comput. Syst. Sci., vol. 78, no. 3, pp. 911-938, 2012.

[9] H. Xu, U. Topcu, and R. M. Murray, "Specification and synthesis of reactive protocols for aircraft electric power distribution," IEEE Trans. Control Netw. Syst., vol. 2, no. 2, pp. 193-203, 2015

[10] N. Bajaj, "Synthesis of Reliable and Cost-Effective Cyber-Physical System Architectures," University of California at Berkeley, 2015.

[11] H. Xu, U. Topcu, and R. M. Murray, "A case study on reactive protocols for aircraft electric power distribution," 2012 IEEE 51st IEEE Conf. Decis. Control, pp. 1124-1129, 2012.

[12] R. Rogersten, H. Xu, N. Ozay, U. Topcu, and R. M. Murray, “An aircraft electric power testbed for validating automatically synthesized reactive control protocols," in Proceedings of the 16th international conference on Hybrid systems: computation and control - HSCC '13, 2013, p. 89.

[13] T. Wongpiromsarn, U. Topcu, N. Ozay, H. Xu, and R. M. Murray, "TuLiP: A Software Toolbox for Receding Horizon Temporal Logic Planning," in Proceedings of the 14th international conference on Hybrid systems: computation and control, 2011, pp. 313-314.

[14] I. Filippidis, S. Dathathri, S. C. Livingston, N. Ozay, and R. M. Murray, "Control design for hybrid systems with TuLiP: The Temporal Logic Planning toolbox," in 2016 IEEE Conference on Control Applications, CCA 2016, 2016, pp. 1030-1041.

[15] A. Pnueli, Y. Sa'ar, and L. D. Zuck, "Jtlv: A framework for developing verification algorithms," Lect. Notes Comput. Sci. (including Subser. Lect. Notes Artif. Intell. Lect. Notes Bioinformatics), vol. 6174 LNCS, pp. 171-174, 2010

[16] A. Pinto and A. L. Sangiovanni Vincentelli, "CSL4P: A Contract Specification Language for Platforms,” Syst. Eng., vol. 20, no. 3, pp. 220-234, May 2017.

[17] J. B. Hu, F. Li, J. Wu, J. H. Wang, and L. P. Zhang, "Research on Aviation Electric Power Cyber Physical Systems," Adv. Mater. Res. vol. 846-847, pp. 126-133, Nov. 2013

[18] A. Benveniste et al., "Contracts for system design," HAL archivesouvertes.fr, 2012.

[19] S. Messaoud, "Optimal Architecture Synthesis for Aircraft Electrical Power Systems,” Technische Universitat Munchen, 2015.

[20] A. Sangiovanni-Vincentelli, W. Damm, and R. Passerone, "Taming Dr. Frankenstein: Contract-Based Design for Cyber-Physical Systems*," Eur. J. Control, vol. 18, no. 3, pp. 217-238, 2012.

[21] P. Nuzzo, J. Finn, M. Mozumdar, and A. Sangiovanni-vincentelli, "Platform-Based Design Methodology and Modeling for Aircraft Electric Power Systems," in Proceedings of Green Energy and Systems Conference, 2013, pp. 1-7.

[22] N. Bajaj, P. Nuzzo, M. Masin, and A. Sangiovanni-Vincentelli, "Optimized Selection of Reliable and Cost-Effective Cyber-Physical System Architectures," in Design, Automation \& Test in Europe Conference \& Exhibition (DATE), 2015, 2015, pp. 561-566.

[23] S. Becz, A. Pinto, L. E. Zeidner, R. Khire, A. Banaszuk, and H. M. Reeve, "Design system for managing complexity in aerospace systems," in 10th AIAA ATIO/ISSMO Conference, 2010, pp. 1-7.

[24] A. Tardy et al., "Towards More Optimization for Aircraft Energy Conversion Systems," in MEA 2015 - More Electric Aircraft -, 2015, no. February, p. 16.

[25] X. Roboam et al., Integrated Design by Optimization of Electrical Energy Systems, 1st ed. UK: John Wiley \& Sons - ISTE, 2012.

[26] B. Wen et al., "Integrated Design by Optimization of Power Systems for More Electric Aircraft," in MEA 2015 - More Electric Aircraft 2015, no. February.
[27] D. R. Vieira, M.-L. Rebaiaia, and M. C. Chain, "The Application of Reliability Methods for Aircraft Design Project Management," Am. J. Ind. Bus. Manag., vol. 06, no. 09, pp. 967-992, 2016.

[28] I. Kaymaz and K. Marti, "Reliability-based design optimization for elastoplastic mechanical structures," Comput. Struct., vol. 85, no. 10, pp. 615-625, 2007.

[29] G. Ezzati, "A reliability-based design optimization model for electricity power networks," in Dynamics of Continuous, Discrete and Impulsive Systems, 1st ed., no. Series B, Applications and Algorithms 22, Watam Press, 2015, pp. 339-357.

[30] Y.-G. Zhao and T. Ono, "A general procedure for first/second-order reliability method (FORM/SORM)," Struct. Saf., vol. 21, no. 2, pp. 95-112, 1999

[31] R. J. T. Montgomery and S. Galloway, "An Optimisation Based Design Approach for Aircraft Electrical Power Systems," SAE Int. J. Aerosp., vol. 7, no. 1, pp. 44-52, 2014.

[32] J. Brombach, A. Lucken, B. Nya, M. Johannsen, and D. Schulz, "Comparison of Different Electrical HVDC- Architectures for Aircraft Application," in Electrical Systems for Aircraft, Railway and Ship Propulsion (ESARS), 2012, 2012, pp. 1-6.

[33] A. Engel, Y. Reich, T. R. Browning, and D. M. Schmidt, "Optimizing system architecture for adaptability," in Proceedings of International Design Conference, DESIGN, 2012, vol. DS 70, pp. 1677-1688.

[34] L. Laguna, R. Prieto, J. A. Oliver, J. A. Cobos, H. Visairo, and P. Kumar, "Power Conversion Modeling Methodology Based on Building Block Models," in Energy Conversion Congress and Exposition (ECCE), 2009 IEEE, 2009, pp. 3404-3410.

[35] L. Laguna, R. Prieto, J. A. Oliver, J. A. Cobos, and H. Visairo-Cruz, "Fast architecture generation and evaluation techniques for the design of large power systems," in 2010 IEEE Energy Conversion Congress and Exposition, 2010, pp. 3464-3469

[36] M. Terörde and D. Schulz, "New real-time heuristics for electrical load rebalancing in aircraft," IEEE Trans. Aerosp. Electron. Syst., vol. 52, no. 3, pp. 1120-1131, 2016

[37] M. Terorde, A. Lucken, and D. Schulz, "Weight saving in the electrical distribution systems of aircraft using innovative concepts," Int. J. energy Res., vol. 31, no. August 2007, pp. 135-147, 2007.

[38] I. Purellku, A. Lucken, J. Brombach, B. Nya, and D. Schulz, "Optimization of the Energy-Supply-Structure of Modern Aircraft By Using Conventional Power System Technologies," in CIRED 21st International Conference on Electricity Distribution, 2011, no. 0973, pp. 6-9.

[39] H. Kim, L. Guo, E. A. Lee, and A. Sangiovanni-Vincentelli, "A Tool Integration Approach for Architectural Exploration of Aircraft EPS with Ptolemy II / Metro II," in IEEE 1st International Conference on Cyber-Physical Systems, Networks, and Applications CPSNA, 2013, no. 1 , pp. $38-43$

[40] Y. Xu, Z. Zhang, J. Li, and Y. Yan, "Architecture analysis and optimization of high voltage DC parallel electric power system for more electric aircraft," in AUS 2016 - 2016 IEEE/CSAA International Conference on Aircraft Utility Systems, 2016, pp. 244-249.

[41] T. L. Magnanti and R. T. Wong, "Network Design and Transportation Planning: Models and Algorithms," Transp. Sci., vol. 18, no. 1, pp. 155,1984

[42] M. Carneiro da Silva, P. Morelato Franca, and P. D. Bishop da Silveira, "Long-range planning of Power Distribution Systems : Secondary Networks," Science (80-. )., vol. 22, no. 3, pp. 179-191, 1996.

[43] X. Roboam, Systemic Design Methodologies for Electrical Energy Systems: Analysis, Synthesis, and Management, 1st ed. Hoboken, New Jersey: John Wiley \& Sons - ISTE, 2012.

[44] S. Ezzati, "Reliability-based Design Optimisation Methods in Largescale Systems," Bull. Aust. Math. Soc., vol. 95, no. 01, pp. 172-173, Feb. 2017.

[45] S. Seong, C. Hu, and S. Lee, "Design under uncertainty for reliable power generation of piezoelectric energy harvester," J. Intell. Mater. Syst. Struct., vol. 28, no. 17, pp. 2437-2449, 2017.

[46] J. Chen, C. Wang, and J. Chen, "Investigation on the selection of electric power system architecture for future more electric aircraft," IEEE Trans. Transp. Electrif., vol. 4, no. 2, pp. 563-576, 2018.

[47] K. N. Areerak, S. V. Bozhko, G. M. Asher, L. De Lillo, and D. W. P. Thomas, "Stability study for a hybrid AC-DC more-electric aircraft power system," IEEE Trans. Aerosp. Electron. Syst., vol. 48, no. 1, pp. 329-347, 2012. 